\title{
Liver transplantation in the critically ill: a multicenter Canadian retrospective cohort study
}

\author{
Constantine J Karvellas ${ }^{1,2^{*}}$, Thomas Lescot ${ }^{3}$, Peter Goldberg ${ }^{3}$, Michael D Sharpe ${ }^{4}$, Juan J Ronco ${ }^{5}$, \\ Eberhard L Renner ${ }^{6}$, Hina Vahidy ${ }^{2}$, Zafrina Poonja ${ }^{2}$, Prosanto Chaudhury ${ }^{7}$, Norman M Kneteman ${ }^{8}$, Markus Selzner $^{6}$, \\ Earl F Cook ${ }^{9}$ and Sean M Bagshaw ${ }^{1}$, for the Canadian Liver Failure Study Group
}

\begin{abstract}
Introduction: Critically ill cirrhosis patients awaiting liver transplantation (LT) often receive prioritization for organ allocation. Identification of patients most likely to benefit is essential. The purpose of this study was to examine whether the Sequential Organ Failure Assessment (SOFA) score can predict 90-day mortality in critically ill recipients of $L T$ and whether it can predict receipt of $L T$ among critically ill cirrhosis listed awaiting LT.

Methods: We performed a multicenter retrospective cohort study consisting of two datasets: (a) all critically-ill cirrhosis patients requiring intensive care unit (ICU) admission before LT at five transplant centers in Canada from 2000 through 2009 (one site, 1990 through 2009), and (b) critically ill cirrhosis patients receiving LT from ICU ( $n=115)$ and those listed but not receiving LT before death $(n=106)$ from two centers where complete data were available.

Results: In the first dataset, 198 critically ill cirrhosis patients receiving LT (mean (SD) age 53 (10) years, 66\% male, median (IQR) model for end-stage liver disease (MELD) 34 (26-39)) were included. Mean (SD) SOFA scores at ICU admission, at 48 hours, and at LT were 12.5 (4), 13.0 (5), and 14.0 (4). Survival at 90 days was $84 \%(n=166)$. In multivariable analysis, only older age was independently associated with reduced 90-day survival (odds ratio (OR), $1.07 ; 95 \% \mathrm{Cl}, 1.01$ to $1.14 ; P=0.013)$. SOFA score did not predict 90 -day mortality at any time. In the second dataset, 47.9\% ( $n=106)$ of cirrhosis patients listed for LT died in the ICU waiting for LT. In multivariable analysis, higher SOFA at 48 hours after admission was independently associated with lower probability of receiving LT $(\mathrm{OR}, 0.89 ; 95 \% \mathrm{Cl}, 0.82$ to $0.97 ; P=0.006)$. When including serum lactate and SOFA at 48 hours in the final model, elevated lactate (at 48 hours) was also significantly associated with lower likelihood of receiving LT $(0.32 ; 0.17$ to $0.61 ; P=0.001)$.

Conclusions: SOFA appears poor at predicting 90-day survival in critically ill cirrhosis patients after LT, but higher SOFA score and elevated lactate 48 hours after ICU admission are associated with a lower probability receiving LT. Older critically ill cirrhosis patients (older than 60) receiving LT have worse 90-day survival and should be considered for LT with caution.
\end{abstract}

\section{Introduction}

Liver transplantation (LT) for established cirrhosis is associated with good outcomes, reaching survival of greater than $80 \%$ at 1 year [1]. However, given the increasing waiting times for LT, some patients deteriorate, precipitating admission to the intensive care unit

\footnotetext{
* Correspondence: dean.karvellas@ualberta.ca

'Division of Critical Care Medicine, Faculty of Medicine and Dentistry, University of Alberta, 3C1.12 Walter C Mackenzie Center, 8440-112 ST NW, Edmonton, Alberta, T6G 2B7, Canada

Full list of author information is available at the end of the article
}

(ICU) [2]. Given the scarcity of organs and costs of prolonged ICU support, being able to discriminate those cirrhosis patients who will maximally benefit from LT after ICU admission would be useful. Therefore, objective measures to score risk of death and major morbidity based on accessible physiological and laboratory values would be useful to guide clinical decision making for liver organ allocation and transplantation among decompensated cirrhosis patients receiving support in a critical care setting [3]. 
Liver-specific scoring systems, such as the Child-Turcotte Pugh (CTP) score and the Model for End-stage Liver Disease (MELD), seem optimal for prognostication in slowly decompensating cirrhosis patients but may not perform as well in those with additional organ dysfunction. The CTP score, validated in cirrhosis patients undergoing surgical esophageal varix ligation/TIPS, includes synthetic hepatic markers (INR, bilirubin, albumin) and complications specific to cirrhosis (ascites and encephalopathy) but does not take into account cardiac, pulmonary, and/or kidney dysfunction (that is, non-liver organ dysfunction) [3-6]. MELD is currently used for organ allocation in North America and has been validated for 3-month survival in cirrhosis patients (all etiologies) without transplant, but its ability to prognosticate outcome with transplant in critically ill cirrhosis patients has not been rigorously evaluated $[7,8]$.

Illness severity scores such as the Acute Physiology and Chronic Health Evaluation (APACHE) II score (see operational definitions) or the Sequential Organ Failure Assessment (SOFA) could potentially offer superior prognostication in the setting of extrahepatic organ dysfunction. APACHE II correlates with hospital outcome in a mixed ICU population and in nontransplanted cirrhosis patients; however, it has been validated for use only at the time of ICU admission [9,10]. SOFA is a validated ICU-specific organ-dysfunction score, developed in a heterogeneous critically ill septic population, and correlates with outcome $[10,11]$. The SOFA score, calculated as a composite of gradations in severity of organ dysfunction across six organ systems (neurologic, respiratory, cardiovascular, renal, hematologic, and hepatic), can be calculated daily to assess evolution of organ dysfunction [11]. Prior studies showed that SOFA scores above 11 correspond to hospital mortality exceeding $80 \%$ in a heterogeneous ICU population [12]. To date; however, the clinical utility of the SOFA score to predict outcomes in critically ill cirrhosis patients has been evaluated only in patients that did not receive LT [13-16].

We hypothesized that increased severity of organ dysfunction, as defined by the SOFA score, would adversely affect 90-day survival and reduce the proportion of listed eligible critically ill cirrhosis patients receiving LT. Accordingly, our objectives were:

1. To determine whether critically ill cirrhosis patients with a high burden of organ dysfunction, as defined by the SOFA score, measured at the time of ICU admission, at 48 hours after admission, and on the day of LT, have a higher 90-day mortality compared with those with less-severe organ dysfunction.

2. To determine whether critically ill cirrhosis patients with a high burden of organ dysfunction, as defined by the SOFA score, measured at the time of ICU admission and at 48 hours after admission, who are eligible for LT, are less likely to receive LT compared with those with less-severe organ dysfunction.

\section{Materials and methods}

The reporting of this study follows the STROBE statement for observational studies [17]. The Health Research Ethics Boards at each participating institution approved this study before commencement. The requirement for individual informed consent was waived.

\section{Design and setting}

For our first objective, we performed a retrospective cohort study of all critically ill cirrhosis patients transplanted $(n=198)$ at five major Canadian liver transplant centers (University of Alberta, Edmonton, Alberta; University of British Columbia, Vancouver, British Columbia; McGill University, Montreal, Quebec; University of Toronto, Toronto, Ontario; and Western University, London, Ontario) between January 2000 and December 2009. Data were collected from the University of Alberta for all cirrhosis patients given transplants between January 1990 and December 2009.

For our second objective, we performed a retrospective cohort study from two LT centers (University of Alberta, Edmonton, from January 1990 through December 2009; McGill University, Montreal, from January 2000 through December 2009) where complete data were available on all critically ill cirrhosis patients who received an LT while in the ICU $(n=115)$ and from those eligible cirrhosis patients listed for LT but who did not receive an LT and died while in the ICU $(n=106)$. Data from the other three centers on eligible critically ill cirrhosis patients listed but who did not receive LT while in the ICU were unavailable.

\section{Participants}

Inclusion criteria were as follows: (a) prior diagnosis of cirrhosis AND listed for LT; (b) age $\geq 18$ years; and (c) admission to an ICU with critical illness/organ failures.

Exclusion criteria were as follows: (a) primary diagnosis of acute (fulminant) liver failure; and (b) liver transplantation from the ward or home; (c) patients receiving retransplant; and (d) multiorgan transplant (for example, liver and kidney)

\section{Operational definitions}

The Sequential Organ Failure Assessment (SOFA) score is an organ-failure scoring system comprising six organsystem domains (neurologic, respiratory, cardiovascular, renal, hematologic, and hepatic) [11]. Each organ system earns a score out of 4 (range, 0 to 4 , with 4 being the worst) and with a maximum score out of 24. The Acute Physiology and Chronic Health Evaluation (APACHE) II 
Score is a illness-severity classification system based on initial values of 12 routine physiologic measurements, age, and previous health status to provide a general measure of severity of disease. An increasing score (range, 0 to 71) correlates with increasing risk of hospital death [10]. The Charlson co-morbidity index (CCI) is a validated summary measure of premorbid chronic disease that correlates with 1-year mortality. The CCI comprises 22 different chronic conditions. Each condition is assigned a score (range, 1, 2, 3, or 6, depending on the severity and the risk of dying associated with this condition) and added to provide a composite score [18]. We used a modified version of this by excluding liver disease. The Donor Risk Index (DRI) is a composite score of donor variables derived from 9,882 deceased LT donors (April 1, 2002 to December 31, 2003) from the Scientific Registry of Transplant Recipients (SRTR) database [19]. Given limited donor information from chart review, we used a modified composite score of variables that were available and based on the DRI (donor age $>60$, partial/split-liver graft, cold ischemia time $>8$ hours, stroke/cerebrovascular accident as principal donor cause of death (either ischemic or hemorrhagic)).

\section{Variables}

Our primary exposure of interest was severity of organ dysfunction, as defined by the SOFA score, sequentially assessed after ICU admission [11]. For the LT cohort (objective $1 ; n=198$ ), the SOFA score was assessed at ICU admission, at 48 hours after admission, and on the day of LT. For the eligible but non-LT cohort (objective 2 ; $n=106)$, the SOFA score was assessed at ICU admission and at 48 hours after admission.

\section{Data sources and collection}

Data were extracted from patient medical records and existing regional liver-transplant databases. Data fields abstracted included the etiology and complications of cirrhosis, preoperative (admission, 48 hours, day of transplant, if applicable) hematologic, biochemical and physiological/organ-dysfunction data (requirement for vasopressors, mechanical ventilation, renal replacement therapy (RRT)), donor information, and outcomes after LT (see Table 1). Data were collected on medical comorbidities (modified CCI) when available. Patient outcomes collected included surgical complications of transplant, duration of mechanical ventilation, duration of ICU/hospital stay, need for postoperative RRT, and mortality.

\section{Statistical analysis \\ First objective: predictors of 90-day mortality after liver transplant (five sites)}

For our first objective, our primary outcome was 90-day mortality among critically ill cirrhosis patients receiving LT.
The rationale for ascertainment of mortality at 90 days was largely to reflect the early perioperative contributing factors (severity of illness, donor characteristics) and to avoid later confounding due to recurrent diseases, such as recurrent hepatitis $\mathrm{C}$ virus (HCV) or graft loss due to noncompliance with immunosuppression. The main covariates examined included age, sex, liver-disease etiology, time of listing to and time from ICU admission to LT, decade of transplant, and graft/donor risk.

In the event of missing values, data were not replaced or estimated. After normality testing of descriptive statistics, normally distributed variables were reported as means with standard deviations (SDs) and compared with the Student $t$ test. Nonnormally distributed continuous data were reported as medians with interquartile ranges (IQRs) and compared with the Wilcoxon ranksum test. Categoric events were compared by using the $\chi^{2}$ test or the Fisher Exact test where appropriate $(n<5$ events). A $P$ value of $<0.05$ was be considered statistically significant for all comparisons. Analysis was performed by using IBM SPSS Version 19 (IBM, New York, USA).

A logistic regression analysis was performed on the cohort of 198 subjects to determine whether the probability of 90-day mortality was affected by SOFA in three separate models (at admission, at 48 hours, and at day of LT). Covariates included in the model were based on univariate unadjusted odds ratios $(P<0.1)$ and predefined covariates; age, sex (female), etiology of liver disease, decade of LT, and transplant site. Etiology was collapsed into hepatitis $\mathrm{C}(\mathrm{HCV})$ versus other, because of the less-favorable outcomes associated with $\mathrm{HCV}$ [20]. To account for changes in ICU/transplant practice, time (decade) was collapsed into a binary variable 1990 through 1999 versus 2000 through 2009, as one site (University of Alberta) had data from 20 years. Covariates in each model were assessed for collinearity. The individual parameters included in SOFA were not included in derivation of these models. Multivariate associations are reported as odds ratios (ORs) with 95\% confidence limits. All presented models met criteria for fit were assessed by the Hosmer-Lemeshow goodnessof-fit test $(P>0.3$ for all).

Second objective: predictors of transplant in 221 critically ill cirrhosis patients (two sites)

For our second objective, physiological data, biochemical data, and SOFA were compared (admission, 48 hours) between all cirrhosis patients transplanted from ICU and those cirrhosis patients listed for LT but who died in the ICU awaiting an organ. The primary outcome was receipt of transplant. Univariable analysis was performed as described as for the first aim.

We subsequently performed a multivariable logistic regression analysis exploring predictors of receipt of LT. Covariates and multivariate associations were reported 
Table 1 Baseline, donor, and outcome characteristics for 198 transplanted critically ill cirrhosis patients (first objective, five sites) and 106 nontransplanted critically ill cirrhosis patients (second objective, two sites)

\begin{tabular}{|c|c|c|}
\hline & First objective $(n=198)$ & Second objective $(n=106)$ \\
\hline Age (years) & $53(10)$ & $54(9.5)$ \\
\hline Female & $67(34 \%)$ & $31(29 \%)$ \\
\hline \multicolumn{3}{|l|}{ Etiology } \\
\hline Hepatitis C & $62(31 \%)$ & $30(29 \%)$ \\
\hline Hepatitis B & $17(9 \%)$ & $7(7 \%)$ \\
\hline Alcohol & $30(15 \%)$ & $24(23 \%)$ \\
\hline $\mathrm{PSC} / \mathrm{PBC}$ & $30(15 \%)$ & $9(9 \%)$ \\
\hline NASH/Cryptogenic & $17(9 \%)$ & $18(17 \%)$ \\
\hline \multicolumn{3}{|l|}{ Comorbidities/Cirrhotic complications } \\
\hline Charlson Score & $1(1)$ & $0.7(1)$ \\
\hline Ascites & $100(96 \%)$ & $61(71 \%)$ \\
\hline Variceal bleeding & $53(56 \%)$ & $54(64 \%)$ \\
\hline Hepatic encephalopathy & 107 (94\%) & $67(79 \%)$ \\
\hline Hepatorenal syndrome & $84(63 \%)$ & 71 (69\%) \\
\hline Spontaneous bacterial peritonitis & $34(41 \%)$ & $37(44 \%)$ \\
\hline \multicolumn{3}{|l|}{ Hematology } \\
\hline Hemoglobin $(g / L)$ & $85(23)$ & $84(22)$ \\
\hline White blood count $\left(\times 10^{9} / \mathrm{L}\right)$ & $8.9(5.2-14.5)$ & $9.7(6.5-15.4)$ \\
\hline Platelet count $\left(\times 10^{9} / \mathrm{L}\right)$ & $64(43-95)$ & $70(40-118)$ \\
\hline \multicolumn{3}{|l|}{ Biochemistry } \\
\hline INR & $2.1(1.7-2.8)$ & $2.2(1.8-3.3)$ \\
\hline ALT (U/L) & $46(25-82)$ & $53(27-128)$ \\
\hline Bilirubin $(\mu M)$ & $273(95-575)$ & $239(95-469)$ \\
\hline Sodium (mM) & $137(130-143)$ & $136(130-143)$ \\
\hline Lactate (mM) & $2.8(1.6-4.6)$ & $3.6(2.4-7.8)$ \\
\hline $\mathrm{pH}$ & $7.39(7.32-7.46)$ & $7.36(7.25-7.44)$ \\
\hline Creatinine $(\mu M)$ & $197(109-308)$ & $207(122-301)$ \\
\hline \multicolumn{3}{|l|}{ Physiology } \\
\hline Mean arterial pressure $(\mathrm{mm} \mathrm{Hg})$ & $67(60-83)$ & $52(10)$ \\
\hline Glasgow Coma Scale score (admission) & $10(5)$ & $9(5)$ \\
\hline $\mathrm{PO}_{2} / \mathrm{FiO}_{2}$ ratio (mm Hg, admission) & $227(106)$ & $195(112)$ \\
\hline \multicolumn{3}{|l|}{ Organ support } \\
\hline Vasopressors (admission) & $84 / 186(45 \%)$ & $54 / 85(64 \%)$ \\
\hline Vasopressors (any day) & 95/108 (88\%) & $58 / 74(78 \%)$ \\
\hline Mechanical ventilation (admission) & 76/134 (57\%) & $50 / 86(58 \%)$ \\
\hline MV (any day) & 100/114 (88\%) & $61 / 74(82 \%)$ \\
\hline RRT (admission) & 49/187 (26\%) & $27 / 87(31 \%)$ \\
\hline RRT (any day) & 78/139 (56\%) & $42 / 76(55 \%)$ \\
\hline \multicolumn{3}{|l|}{ Aggregate scores } \\
\hline Child Turcotte Pugh (listing) & $12.4(1.6)$ & \\
\hline MELD (listing) & $24(16-36)$ & $23(15-35)$ \\
\hline MELD (admit) & $34(26-39)$ & $36(27-40)$ \\
\hline MELD (transplant) & $34(27-40)$ & \\
\hline SOFA (admit) & $12.5(4)$ & $14(4)$ \\
\hline SOFA (48 hours) & $13(5)$ & $17(4)$ \\
\hline SOFA (transplant) & $14(4)$ & \\
\hline
\end{tabular}


Table 1 Baseline, donor, and outcome characteristics for 198 transplanted critically ill cirrhosis patients (first objective, five sites) and 106 nontransplanted critically ill cirrhosis patients (second objective, two sites) (Continued)

\begin{tabular}{ll}
\hline Mortality & $32(16 \%)$ \\
\hline 90 days & $52(26 \%)$ \\
1 year & $63(38 \%)$ \\
3 years & \\
\hline Donor characteristics & $35(18 \%)$ \\
\hline Donor age $>60$ & $2(1 \%)$ \\
Partial graft (split) & $105(54 \%)$ \\
Donor cerebrovascular accident & $102(56 \%)$ \\
Cold ischemia time $>8$ hours & $1(1-2)$ \\
Donor score (of 4$)$ &
\end{tabular}

Charlson score was modified to exclude liver disease.

as described for the first aim. From univariate logistic regression $(P$ value of $<0.10)$, lactate was included with predefined covariates. Lactate (admission at 48 hours) was transformed into its natural logarithm to meet the assumption of a normal distribution.

\section{Results}

First objective: predictors of mortality in transplanted critically ill cirrhosis patients

Participants and descriptive data

In total, 198 critically ill cirrhosis patients (mean (SD) age, 53 (10) years, 66\% male) received transplants during the study period. Baseline characteristics are shown in Table 1. The most common etiologies were hepatitis $\mathrm{C}$ (31\%) and alcohol abuse (15\%). LT occurred a median (IQR) of 29 (5 to 101) days from listing and 5 (3 to 10) days from ICU admission. While in the ICU, $88 \%$ received vasopressors, $56 \%$ received RRT, and $87 \%$ were mechanically ventilated before $\mathrm{LT}$. The median (IQR) MELD score was 34 ( 26 to 39 ) on ICU admission and 34 (27 to 40 ) on the day of LT, respectively. SOFA scores (mean (SD)) were 12.5 (4), 13 (5), and 14 (4) on ICU admission, at 48 hours, and on the day of LT, respectively.

Overall, 166 (84\%) of all 198 transplanted critically ill cirrhosis patients were alive at 90 days, 145 (74\%) of 197 at 1 year, and 105 (62.5\%) of 163 at 3 years. Sixteen (8\%) patients were given repeated transplants. Donor characteristics are also shown in Table 1 . Thirty-five (18\%) of 193 patients received a graft from a donor aged $>60$ years, $1 \%$ received a split graft, $54 \%$ of donors died of a cerebrovascular accident (ischemic or hemorrhagic), and $56 \%$ of donor organs were transplanted with a coldischemia time exceeding 8 hours.

\section{Univariable outcome data: 198 liver transplant recipients}

Results of univariable analysis are shown in Table 2. Comparing patients who were alive $(n=166,84 \%)$ with those who were dead $(n=32,16 \%)$ at 90 days, no statistically significant differences in MELD were found on admission or day of LT ( $P>0.6$ for both). No statistically significant differences were seen between SOFA on admission or day of LT ( $P>0.17$ for both). Patients alive at 90 days were significantly younger (51 versus 56 years; $P=0.007)$. Patients older than 60 years had significantly higher 90 -day mortality ( $27 \%$ versus $13 \%$; $P=$ $0.04)$, and a trend toward increased 1-year mortality (37\% versus $23 \% ; P=0.09$ ). No significant differences in donor characteristics were noted comparing LT recipients alive at 90 days with nonsurvivors. The primary cause of death in patients deceased $<90$ days was sepsis/ multiorgan failure $(n=7,54 \%$ where data were available).

\section{Multivariable analysis: predictors of mortality}

Three separate multivariable logistic regression models were built to assess the impact of the exposure SOFA on admission (model 1), SOFA 48 hours after ICU admission (model 2), and SOFA on the day of LT (model 3) and survival (Table 3). After covariate adjustment, SOFA was not significantly predictive of 90-day mortality when assessed at ICU admission, at 48 hours, or on the day of LT ( $P>0.2$ for all). In all three models, age (per incremental year) was independently associated with higher 90 -day mortality in all models $(P<0.03$ for all models) after controlling for SOFA, sex, etiology, decade of transplant, and transplant center.

Second objective: predictors of transplant in 221 critically ill cirrhosis patients (two sites)

\section{Participants and descriptive data}

Baseline data of 106 nontransplanted cirrhosis patients are shown in Table 1 . The most common etiologies were hepatitis C (29\%) and alcohol abuse (23\%). In total, $78 \%$ required vasopressors, $55 \%$ received RRT, and $82 \%$ were mechanically ventilated while in the ICU. The median (IQR) MELD score was 36 (27 to 40) on ICU admission. Mean (SD) SOFA scores were 14 (4) and 17 (4) on ICU admission and at 48 hours, respectively. 
Table 2 Univariable analysis of admission and pretransplant factors for survivors versus nonsurvivors at 90 days in 198 transplanted critically ill cirrhosis patients (five sites)

\begin{tabular}{|c|c|c|c|}
\hline & Alive at 90 days $(n=166)$ & Dead at 90 days $(n=32)$ & $P$ value \\
\hline Age & $51(11)$ & $56(8)$ & 0.007 \\
\hline Age $>60$ & $30(18 \%)$ & $11(34 \%)$ & 0.037 \\
\hline Female & $58 / 165$ (35\%) & 9/32 (28\%) & 0.4 \\
\hline $\mathrm{HCV}$ & $54 / 166(33 \%)$ & $8 / 32(25 \%)$ & 0.4 \\
\hline \multicolumn{4}{|l|}{ Biochemistry (admission) } \\
\hline Hemoglobin ( $g / L)$ & $85(24)$ & $86(21)$ & 0.9 \\
\hline WBC $\left(\times 10^{9} / \mathrm{L}\right)$ & $9.2(5.2-14.8)$ & $8.0(5.5-11.8)$ & 0.4 \\
\hline Platelets $\left(\times 10^{9} / L\right)$ & $62(41-94)$ & $71(49-109)$ & 0.14 \\
\hline INR & $2.1(1.7-2.7)$ & $2.4(1.8-3.0)$ & 0.3 \\
\hline Bilirubin $(\mu M)$ & $272(101-589)$ & $269(74-540)$ & 0.4 \\
\hline Lactate (mM) & $2.8(1.7-4.6)$ & $1.5(1.1-4.6)$ & 0.23 \\
\hline Sodium (mM) & $136(131-142)$ & $136(131-141)$ & 0.7 \\
\hline Creatinine $(\mu M)$ & $195(97-303)$ & $225(141-336)$ & 0.17 \\
\hline \multicolumn{4}{|l|}{ Physiology (admission) } \\
\hline GCS & $10(4.5)$ & $11(4.5)$ & 0.6 \\
\hline $\mathrm{PO}_{2} / \mathrm{FiO}_{2}$ ratio $(\mathrm{mm} \mathrm{Hg})$ & $229(102)$ & $217(132)$ & 0.5 \\
\hline Mechanical ventilation & $68 / 121(56 \%)$ & $8 / 13(62 \%)$ & 0.7 \\
\hline Vasoactive drugs & 75/157 (48\%) & $9 / 29(31 \%)$ & 0.10 \\
\hline Renal replacement therapy & 43/158 (27\%) & $6 / 29(21 \%)$ & 0.5 \\
\hline \multicolumn{4}{|l|}{ Child-Turcotte-Pugh score } \\
\hline Listing & $12.5(1.4)$ & $12.9(1.4)$ & 0.5 \\
\hline \multicolumn{4}{|l|}{ MELD } \\
\hline Listing & $24(16-36)$ & $27(17-27)$ & 0.85 \\
\hline Admission & $34(26-39)$ & $34(25-40)$ & 0.6 \\
\hline Day of transplant & $35(26-40)$ & $34(28-40)$ & 0.9 \\
\hline \multicolumn{4}{|l|}{ SOFA } \\
\hline Admission & $13(4)$ & $12(5)$ & 0.11 \\
\hline 48 hours & $14(5)$ & $11(4)$ & 0.023 \\
\hline Day of transplant & $14(4)$ & $11(4)$ & 0.15 \\
\hline \multicolumn{4}{|l|}{ ICU Length of stay } \\
\hline Before transplant & $5(3-10)$ & $6(2-14)$ & 0.6 \\
\hline After transplant & $9(5-20)$ & $8(2-14)$ & 0.05 \\
\hline Donor score (out of 4) & $1(1-2)$ & $1(1-2)$ & 0.6 \\
\hline Partial graft & $2 / 160(1 \%)$ & $0 / 30(0)$ & 0.5 \\
\hline Cold ischemia time $>8$ hours & $88 / 157(56 \%)$ & $14 / 26(54 \%)$ & 0.8 \\
\hline Donor cerebrovascular accident & $85 / 163(52 \%)$ & $20 / 30(67 \%)$ & 0.14 \\
\hline Donor age $>60$ & 29/162 (18\%) & 6/31 (19\%) & 0.8 \\
\hline
\end{tabular}

\section{Univariable analysis: Liver transplant in 221 critically ill cirrhotics (two sites)}

Patients who died waiting for LT had higher MELD scores on admission (36 versus $33, P=0.035$; see Additional file 1). Furthermore, SOFA on admission (14 versus $13 ; P=0.048$ ) and at 48 hours after ICU admission (17 versus $13 ; P<0.001$ ) were significantly higher in patients who went on not to receive LT (See Figure 1). On ICU admission, patients who died waiting for LT had worse coagulopathy (INR 2.2 versus $2.0 ; P=0.009$ ), acidemia ( $\mathrm{pH} 7.36$ versus $7.38 ; P=0.019)$ and had higher serum lactate levels ( 3.6 versus $2.6 ; P=0.003$ ). On admission, non-LT patients were more likely to be hypotensive (MAP, 54 versus 66; $P<0.001$ ) and to require vasopressor support (64\% versus $47 \% ; P=0.02)$.

\section{iii) Multivariable analysis: predictors of transplant}

\section{(two sites)}

After covariate-adjustment, SOFA on admission was not significantly associated with receipt of LT (OR, 0.96; 95\% CI, 0.89 to 1.04; see Additional file 2). In multivariable 
Table 3 Multivariable analysis: predictors of 90-day mortality in 198 critically ill cirrhosis patients who underwent liver transplantation

\begin{tabular}{lllll}
\hline Covariate & Unadjusted & Model $\mathbf{1}(\boldsymbol{n}=\mathbf{1 8 0})$ & Model 2 $(\boldsymbol{n}=\mathbf{1 1 0})$ & Model $\mathbf{3}(\boldsymbol{n}=\mathbf{1 4 0})$ \\
\hline Age & $1.06(1.01-1.11)^{\mathrm{a}}$ & $1.07(1.01-1.14)^{\mathrm{b}}$ & $1.12(1.02-1.22)^{\mathrm{c}}$ & $1.07(1.01-1.14)^{\mathrm{d}}$ \\
\hline Gender (female) & $0.72(0.31-1.66)$ & $0.72(0.29-2.39)$ & $0.24(0.05-1.181)$ & $0.60(0.19-1.82)$ \\
\hline Etiology (HCV versus non-HCV) & $0.69(0.29-1.64)$ & $0.86(0.30-2.40)$ & $0.39(0.100-1.181)$ & $1.07(0.37-3.07)$ \\
\hline \hline SOFA (admission) & $0.93(0.85-1.03)$ & $1.07(0.94-1.22)$ & & \\
\hline SOFA (48 hours) & $0.88(0.79-0.99)$ & & $1.04(0.87-1.23)$ & $1.04(0.90-1.19)$ \\
\hline SOFA (day of transplant) & $0.94(0.85-1.04)$ & & $35.73(8)$ & $23.81(8)$ \\
\hline \hline$\chi^{2}$ (degrees of freedom) & & $31.00(9)$ & & \\
\hline
\end{tabular}

All three multivariable models were adjusted for site (five) and decade of transplant (2000 through 2009 versus 1990 through 1990$)$. Hosmer-Lemeshow Goodness of Fit performed well $(P>0.15)$ for all models. Model one included age, gender (female), etiology (HCV versus non-HCV) and SOFA on admission ( $n=180 / 198$ patients; 18 patients had missing data). Model two had similar covariates but incorporated SOFA at 48 hours after admission ( $n=110 ; 88$ patients had missing data). Model three had similar covariates but incorporated SOFA on the day of liver transplant $(n=140 ; 58$ patients had missing data). Significant $P$ values were for age (unadjusted $(P=0.013),{ }^{a}$ Model $1(P=0.015),{ }^{\text {b }}$ Model $2(P=0.014)$, and ${ }^{\mathrm{d}}$ Model $3(P=0.027)$.

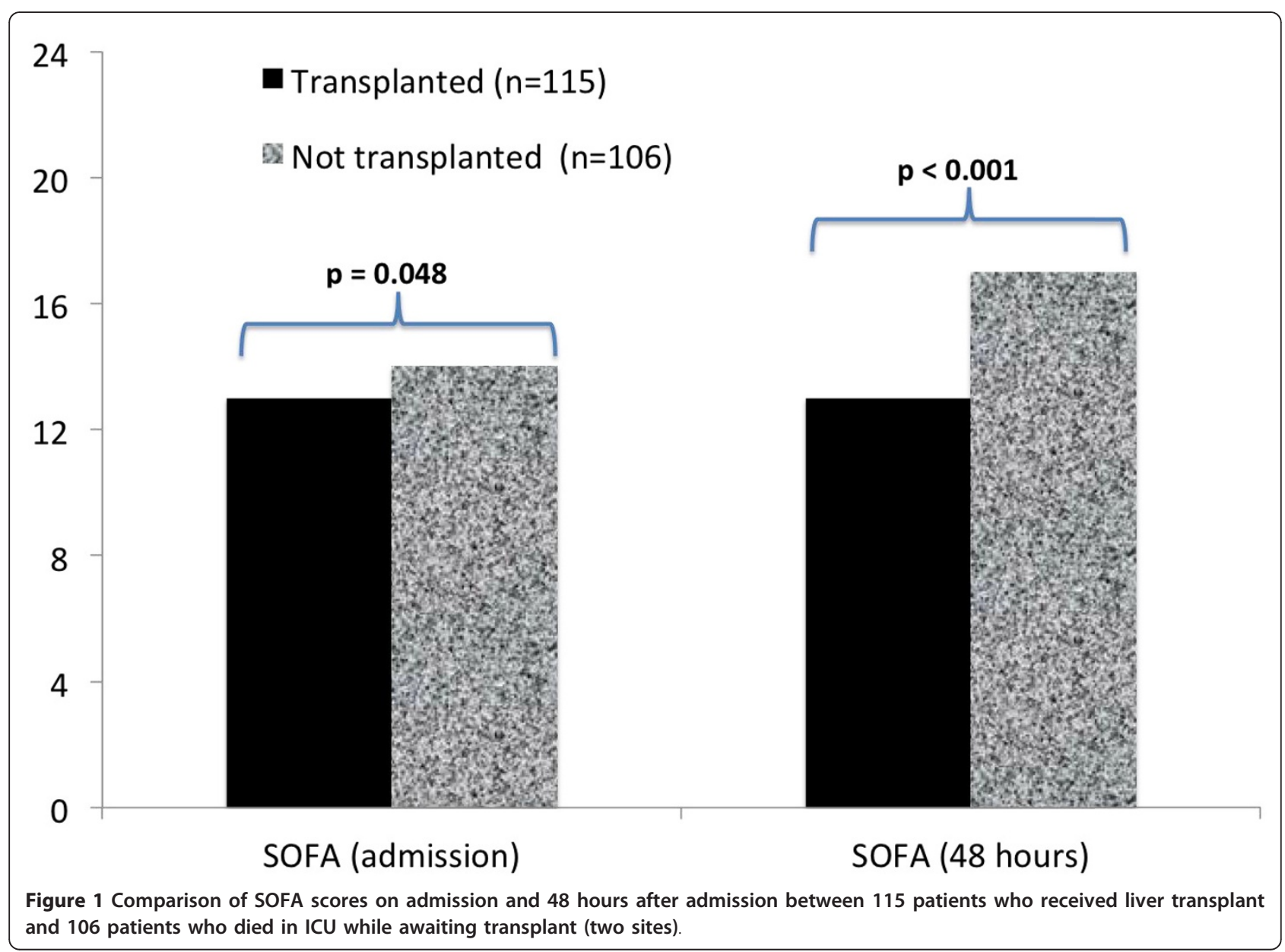

analysis of SOFA at 48 hours after admission, after adjustment for patient age and other covariates except lactate, higher SOFA at 48 hours was associated with a lower likelihood of receiving LT (see Table 4: OR, $0.89 ; 95 \% \mathrm{CI}, 0.82$ to $0.97 ; P=0.006)$. However when lactate at 48 hours is included in the model with other covariates, 48-hour lactate appears to have a stronger association with likelihood of transplant (OR, 0.32; 95\% CI, 0.17 to $0.61 ; P=0.001)$ compared with SOFA $(P=0.19)$. 
Table 4 Multivariable analysis: predictors of receipt of liver transplant in 221 critically ill cirrhosis patients on transplant list in ICU

\begin{tabular}{|c|c|c|c|c|}
\hline Covariate & Unadjusted $(n=221)$ & Model $1(n=126)$ & Model $2(n=174)$ & Model $3(n=125)$ \\
\hline Age & $0.99(0.96-1.01)$ & $0.95(0.91-0.99)^{a}$ & $0.98(0.94-1.01)$ & $0.95(0.91-0.99)^{a}$ \\
\hline Gender (female) & $1.06(0.59-1.89)$ & $1.51(0.64-3.57)$ & $1.02(0.51-2.05)$ & $1.58(0.66-3.80)$ \\
\hline Etiology (HCV versus non-HCV) & $1.28(0.72-2.28)$ & $1.78(0.76-4.14)$ & $1.63(0.81-3.28)$ & $1.72(0.73-4.03)$ \\
\hline SOFA (admission) ${ }^{\mathrm{b}}$ & \multicolumn{4}{|l|}{$0.94(0.88-0.99)^{a}$} \\
\hline Lactate (admission, natural $\log )^{c}$ & \multicolumn{4}{|l|}{$0.52(0.34-0.79)^{a}$} \\
\hline SOFA (48 hours) & \multicolumn{2}{|l|}{$0.88(0.82-0.94)^{a}$} & $0.89(0.82-0.97)^{a}$ & $0.92(0.82-1.04)$ \\
\hline 48-hour lactate (natural log) & $0.30(0.17-0.53)^{a}$ & \multicolumn{2}{|l|}{$0.29(0.15-0.53)^{a}$} & $0.32(0.17-0.61)^{a}$ \\
\hline \multicolumn{2}{|l|}{$\chi^{2}$ (degrees of freedom) } & $31.03(6)$ & $23.75(6)$ & $33.23(7)$ \\
\hline
\end{tabular}

\section{Discussion}

We performed a retrospective cohort study from the five largest liver-transplant centers in Canada to evaluate the impact of critical illness and organ dysfunction on short-term mortality after LT and on organ allocation among critically ill cirrhosis patients listed for LT and admitted to the ICU.

\section{Key results}

We found that among critically ill cirrhosis patients who received LT, older age independently portends a significantly higher risk for post-LT 90-day mortality. We also showed that SOFA score on ICU admission, at 48 hours, and on the day of LT is not predictive of 90-day mortality. When comparing those patients who received LT from the ICU with those patients who died in the ICU awaiting LT, we found that SOFA at 48 hours after ICU admission is independently associated with a lower likelihood of receiving LT. We also found that high lactate (on admission and at 48 hours) and patient age are independently associated with lower likelihood for LT.

\section{Comparison with previous studies}

This study extends on data from previous studies as it is a multicenter study across an organ-sharing network (Canada) and specifically focused on critically ill cirrhosis patients who were listed or received LT while admitted to an ICU. In a Swiss observational study of 144 consecutive LT recipients, Oberkofler and colleagues [21] showed that a MELD >23 was predictive of increased postoperative length of ICU stay but not lower short-term survival [21]. This study, however, was limited because of being representative of a single-center study and including a heterogeneous cohort of ICU and non-ICU patients receiving LT. Given that non-ICU patients were included, only MELD (not SOFA) could be evaluated. Nonetheless, this study showed no association between MELD and post-LT outcome in the critically ill. Previously, Cholangitas and colleagues [22] showed that, in nontransplanted cirrhosis patients from a single center, prognostic scores had increased sensitivity at 48 hours rather than on admission and that SOFA outperformed MELD and APACHE II (on admission). However, this study did not directly evaluate outcomes of critically ill cirrhosis patients receiving LT from the ICU or predictors of receiving an LT. In another singlecenter Taiwanese study, Wong et al. [23] found that although the pre-LT SOFA score predicted unadjusted 3-month and 1-year survival, not all patients were critically ill and admitted to the ICU before LT, and no covariate adjustment was performed [23]. Although SOFA did not appear to be independently associated with 90-day survival in critically ill transplanted cirrhosis patients, our data did show that a higher SOFA score 48 hours after ICU admission was independently associated with a lower likelihood of receiving LT. In contrast with a single-center study from Umgelter and colleagues [24] that reported overall 90 -day mortality rates of almost $40 \%$ for transplanted critically ill cirrhosis patients $(n=23)$, in our cohort, 90-day mortality was lower (16\%).

\section{Study limitations}

Our study has several limitations that warrant consideration. First, although our study is multicentric and represents five major liver-transplant centers in Canada (data not available from two other centers), it is relatively small, and a retrospective analysis of prospectively collected data is thereby potentially predisposed to selection bias and residual confounding. Second, individual centers vary in volume and make independent decisions about listing for LT; hence variations may occur in listing/de-listing practices across sites. However, the 
evaluation of health outcomes, such as organ allocation in critically ill cirrhosis patients and their associated LT outcomes to date has not been evaluated in a prospective manner. Furthermore, all transplant centers that provided data for this study participate in the Canadian Liver transplant organ sharing network, in which decisions are often made to transfer organs from different regions across the country for critically ill cirrhosis patients requiring LT [25]. Accordingly, replicating observational studies of this nature has value to assess for consistency and generalizability. Third, although we attempted to collect data on the donor risk index [19], our data were not complete for all patients, and we used a modified donor-risk score. Furthermore, we were unable to obtain reliable data on surgical complications and intraoperative parameters from all sites.

Finally, we acknowledge that age, as an independent predictor of increased 90-day mortality, could potentially be confounded by virtue of being a surrogate of increased comorbidity [26]. However, as opposed to other critically ill populations, LT candidates are often excluded for having significant cardiopulmonary morbidity or coexisting malignancy. Low Charlson comorbidity scores in this cohort reflected this.

In this study, we found that SOFA was not independently associated with short-term mortality after LT. Because of the complexities of decision making in LT and the inherent selection bias (that is, selected centers may inherently decline sicker patients), it remains to be determined whether SOFA is the best organ-failure score with which to prognosticate in this cohort $[13,22]$. Given the limitation of retrospective studies, future research should be focused on well-designed prospective studies looking at reliable collection of multiple confounding variables. With adequate patient numbers for derivation and validation of a prediction rule, a novel dynamic scoring system that includes other factors (for example, age, lactate) that are not incorporated into the SOFA score could be derived to reflect accurately characteristics that may not be shared between critically ill patients listed for LT and other critically ill populations. Important outcomes, besides mortality, also include the impact of critical illness on health-service allocation issues, such as length of time on mechanical ventilation/ renal replacement therapy, rehospitalization, and persistent nonliver organ dysfunction (neurocognitive, respiratory, nutritional issues). Organ dysfunction has been previously shown to affect cost significantly in critically ill nontransplanted cirrhosis patients, and it would be useful to look at the impact on transplant recipients [27].

\section{Conclusions}

SOFA does not appear to predict outcome after LT in this cohort. However, higher SOFA scores at 48 hours after admission, along with older age and serum lactate, are associated with a lower probability of receiving LT. Older critically ill cirrhosis patients (older than 60) undergoing LT have significantly worse 90-day post-LT mortality and should be considered for transplant with caution.

\section{Key messages}

- SOFA does not appear to predict outcome after LT in critically ill cirrhosis patients

- Older age is significantly associated with worse early outcomes after LT in critically ill cirrhosis patients

- Higher SOFA scores at 48 hours after ICU admission are associated with a lower probability of receiving $\mathrm{LT}$ in critically ill cirrhosis patients listed for LT.

- Higher serum lactate at 48 hours after ICU admission is associated with a lower probability of receiving LT in critically ill cirrhosis patients listed for LT.

\section{Additional material}

Additional file 1: Univariable analysis comparing 115 transplanted cirrhosis patients with106 cirrhosis patients listed but who died while waiting for transplant (two sites). Demographic, biochemical, and physiological comparisons between transplanted and nontransplanted cirrhosis patients (unadjusted).

Additional file 2: Multivariable analysis: Predictors of receipt of liver transplant in 221 critically ill cirrhosis patients on the transplant list in the ICU.Description: Multivariable (adjusted) predictors of liver transplantation (2 sites).

\section{Abbreviations}

AoCLF: Acute-on-chronic liver failure; APACHE: Acute Physiology and Chronic Health Evaluation; CCl: Charlson Comorbidity Index; HBV: hepatitis B; HCV: hepatitis C; ICU: intensive care unit; LT: liver transplant; MELD: Model for Endstage Liver Disease; MV: mechanical ventilation; PNF: primary nonfunction; RRT: renal replacement therapy; SOFA: Sequential Organ Failure Assessment.

\section{Authors' contributions}

CJK wrote and extensively revised the manuscript, compiled the final database, and performed statistical and data analyses. TL, PG, MDS, JJR, ELR, NMK, PC, and MS provided content expertise and assisted in compiling the database, manuscript editing, and revision. HV and ZP assisted in compiling the database and manuscript revision. EFC was a statistical advisor for the study and assisted with significant manuscript revision. SMB provided content expertise, significant guidance on analysis and interpretation of data and assisted extensively with manuscript revision. All authors read and approved the final manuscript for publication.

\section{Competing interests}

The authors declare that they have no competing interests.

\section{Acknowledgements}

The Canadian Liver Failure Study Group comprises the following collaborators: Tracey Bentall (Western University), Prosanto Chaudhury (McGill University), E. Francis Cook (Harvard School of Public Health), Denise Foster (University of British Columbia), Peter Goldberg (McGill University), Constantine J. Karvellas (University of Alberta), Norman M. Kneteman (University of Alberta), Thomas Lescot (McGill University) Max Marquez 
(University of Toronto), Glenda Meeberg (University of Alberta), Peter Metrakos (McGill University), Zafrina Poonja (University of Alberta), Eberhard L. Renner (University of Toronto), Juan J. Ronco (University of British Columbia), Markus Selzner (University of Toronto), Michael D. Sharpe (Western University), Puneeta Tandon (University of Alberta), and Hina Vahidy (University of Alberta).

The study was sponsored by a grant from the Alberta Transplant Fund. Dr. Bagshaw is supported by a Canada Research Chair in Critical Care Nephrology and a Clinician Scientist Award from Alberta Innovates-Health Solutions (AlHS). Dr. Lescot was supported by research fellowships from La Fondation des Gueules Cassées (FGC; Paris, France) and Assistance Publique-Hôpitaux de Paris (AP-HP; Paris, France). CIHR, Al-HS, FGC or AP-HP did not have any involvement in the design and conduct of the study.

Special thanks to Jack Yeung for assistance with database preparation and merging.

\section{Author details}

'Division of Critical Care Medicine, Faculty of Medicine and Dentistry, University of Alberta, 3C1.12 Walter C Mackenzie Center, 8440-112 ST NW, Edmonton, Alberta, T6G 2B7, Canada. ${ }^{2}$ Division of Hepatology, Department of Medicine, Faculty of Medicine and Dentistry, University of Alberta, 130 University Campus NW, Edmonton, Alberta, T6G-2X8, Canada. ${ }^{3}$ Division of Critical Care Medicine, McGill University, McGill University Health Center, 687 Pine Avenue West, Montreal, Quebec, H3A 1A1, Canada. ${ }^{4}$ Division of Critical Care Medicine, Western University, London, Ontario, Canada. ${ }^{5}$ Division of Critical Care Medicine, University of British Columbia, Vancouver General Hospital, 855 West 12th Avenue, Vancouver, British Columbia, V5Z 1M9, Canada. ${ }^{6}$ Multi-Organ Transplant Program, University Health Network/ University of Toronto, 585 University Avenue, Toronto, Ontario, M5G 2N2, Canada. ${ }^{7}$ Division of Solid Organ Transplantation, McGill University Health Center, 687 Pine Avenue West, Montreal, Quebec, H3A 1A1, Canada. ${ }^{8}$ Division of Transplantation, Department of Surgery, University of Alberta, 2D4.44 Walter C. Mackenzie Centre, Edmonton, Alberta, T6G 2B7, Canada. ${ }^{9}$ Department of Epidemiology, Harvard School of Public Health, 677 Huntington Avenue, Boston, Massachusetts 02115, USA

Received: 13 August 2012 Revised: 16 November 2012 Accepted: 25 January 2013 Published: 9 February 2013

\section{References}

1. Futagawa Y, Terasaki PI, Waki K, Cai J, Gjertson DW: No improvement in long-term liver transplant graft survival in the last decade: an analysis of the UNOS data. Am J Transplant 2006, 6:1398-1406.

2. Zimmerman JE, Wagner DP, Seneff MG, Becker RB, Sun $X$, Knaus WA: Intensive care unit admissions with cirrhosis: risk-stratifying patient groups and predicting individual survival. Hepatology 1996, 23:1393-1401.

3. Tsai MH, Chen YC, Ho YP, Fang JT, Lien JM, Chiu CT, Liu NJ, Chen PC: Organ system failure scoring system can predict hospital mortality in critically ill cirrhotic patients. J Clin Gastroenterol 2003, 37:251-257.

4. Pugh RN, Murray-Lyon IM, Dawson JL, Pietroni MC, Williams R: Transection of the oesophagus for bleeding oesophageal varices. Br J Surg 1973, 60:646-649.

5. Shellman RG, Fulkerson WJ, DeLong E, Piantadosi CA: Prognosis of patients with cirrhosis and chronic liver disease admitted to the medical intensive care unit. Crit Care Med 1988, 16:671-678.

6. Castera L, Pauwels A, Levy VG: [Prognostic indicators in patients with liver cirrhosis admitted to an intensive care unit]. Gastroenterol Clin Biol 1996, 20:263-268.

7. Kamath PS, Wiesner RH, Malinchoc M, Kremers W, Therneau TM Kosberg CL, D'Amico G, Dickson ER, Kim WR: A model to predict survival in patients with end-stage liver disease. Hepatology 2001, 33:464-470.

8. Wiesner R, Edwards E, Freeman R, Harper A, Kim R, Kamath P, Kremers W, Lake J, Howard T, Merion RM, Wolfe RA, Krom R, United Network for Organ Sharing Liver Disease Severity Score Committee: Model for end-stage liver disease (MELD) and allocation of donor livers. Gastroenterology 2003, 124:91-96

9. Karvellas CJ, Pink F, McPhail M, Austin M, Auzinger G, Bernal W, Sizer E, Kutsogiannis DJ, Eltringham I, Wendon JA: Bacteremia, acute physiology and chronic health evaluation II and modified end stage liver disease are independent predictors of mortality in critically ill nontransplanted patients with acute on chronic liver failure. Crit Care Med 2009, [Au Query: Please provide volume and pages.].

10. Knaus WA, Draper EA, Wagner DP, Zimmerman JE: APACHE II: a severity of disease classification system. Crit Care Med 1985, 13:818-829.

11. Vincent JL, Moreno R, Takala J, Willatts S, De Mendonca A, Bruining H, Reinhart CK, Suter PM, Thijs LG: The SOFA (Sepsis-related Organ Failure Assessment) score to describe organ dysfunction/failure; on behalf of the Working Group on Sepsis-Related Problems of the European Society of Intensive Care Medicine. Intensive Care Med 1996, 22:707-710.

12. Ferreira FL, Bota DP, Bross A, Melot C, Vincent JL: Serial evaluation of the SOFA score to predict outcome in critically ill patients. JAMA 2001, 286:1754-1758.

13. Cholongitas E, Senzolo M, Patch D, Kwong K, Nikolopoulou V, Leandro G, Shaw S, Burroughs AK: Risk factors, sequential organ failure assessment and model for end-stage liver disease scores for predicting short term mortality in cirrhotic patients admitted to intensive care unit. Aliment Pharmacol Ther 2006, 23:883-893.

14. Ho YP, Chen YC, Yang C, Lien JM, Chu YY, Fang JT, Chiu CT, Chen PC, Tsai $\mathrm{MH}$ : Outcome prediction for critically ill cirrhotic patients: a comparison of APACHE II and Child-Pugh scoring systems. J Intensive Care Med 2004, 19:105-110.

15. Chen YC, Tian YC, Liu NJ, Ho YP, Yang C, Chu YY, Chen PC, Fang JT, Hsu CW, Yang CW, Tsai MH: Prospective cohort study comparing sequential organ failure assessment and acute physiology, age, chronic health evaluation III scoring systems for hospital mortality prediction in critically ill cirrhotic patients. Int J Clin Pract 2006, 60:160-166.

16. Wehler M, Kokoska J, Reulbach U, Hahn EG, Strauss R: Short-term prognosis in critically ill patients with cirrhosis assessed by prognostic scoring systems. Hepatology 2001, 34:255-261.

17. von Elm E, Altman DG, Egger M, Pocock SJ, Gotzsche PC, Vandenbroucke JP: Strengthening the Reporting of Observational Studies in Epidemiology (STROBE) statement: guidelines for reporting observational studies. BMJ 2007, 335:806-808.

18. Charlson ME, Pompei P, Ales KL, MacKenzie CR: A new method of classifying prognostic comorbidity in longitudinal studies: development and validation. J Chronic Dis 1987, 40:373-383.

19. Feng S, Goodrich NP, Bragg-Gresham JL, Dykstra DM, Punch JD, DebRoy MA, Greenstein SM, Merion RM: Characteristics associated with liver graft failure: the concept of a donor risk index. Am J Transplant 2006, 6:783-790.

20. Thuluvath PJ, Maheshwari A, Thuluvath NP, Nguyen GC, Segev DL: Survival after liver transplantation for hepatocellular carcinoma in the model for end-stage liver disease and pre-model for end-stage liver disease eras and the independent impact of hepatitis C virus. Liver Transplant 2009, 15:754-762.

21. Oberkofler CE, Dutkowski P, Stocker R, Schuepbach RA, Stover JF, Clavien PA, Bechir M: Model of end stage liver disease (MELD) score greater than 23 predicts length of stay in the ICU but not mortality in liver transplant recipients. Critical Care 2010, 14:R117.

22. Cholongitas E, Betrosian A, Senzolo M, Shaw S, Patch D, Manousou P, O'Beirne J, Burroughs AK: Prognostic models in cirrhotics admitted to intensive care units better predict outcome when assessed at $48 \mathrm{~h}$ after admission. J Gastroenterol Hepatol 2008, 23:1223-1227.

23. Wong CS, Lee WC, Jeng CC, Tian YC, Chang MY, Lin CY, Fang JT, Yang CW, Tsai MH, Shih HC, Chen YC: Scoring short-term mortality after liver transplantation. Liver Transplant 2010, 16:138-146.

24. Umgelter A, Lange $K$, Kornberg A, Buchler P, Friess H, Schmid RM: Orthotopic liver transplantation in critically ill cirrhotic patients with multi-organ failure: a single-center experience. Transplant Proc 2011, 43:3762-3768.

25. Mullen MA, Kohut N, Sam M, Blendis L, Singer PA: Access to adult liver transplantation in Canada: a survey and ethical analysis. CMAJ 1996, 154:337-342.

26. Schneeweiss S, Maclure M: Use of comorbidity scores for control of confounding in studies using administrative databases. Int J Epidemiol 2000, 29:891-898.

27. Shawcross DL, Austin MJ, Abeles RD, McPhail MJ, Yeoman AD, Taylor NJ, Portal AJ, Jamil K, Auzinger G, Sizer E, Bernal W, Wendon JA: The impact of organ dysfunction in cirrhosis: survival at a cost? J Hepatol 2012, 56:1054-1062. 


\section{doi:10.1186/cc12508}

Cite this article as: Karvellas et al: Liver transplantation in the critically

ill: a multicenter Canadian retrospective cohort study. Critical Care 2013

$17: R 28$.

Submit your next manuscript to BioMed Central and take full advantage of:

- Convenient online submission

- Thorough peer review

- No space constraints or color figure charges

- Immediate publication on acceptance

- Inclusion in PubMed, CAS, Scopus and Google Scholar

- Research which is freely available for redistribution 